\title{
Hydrazone-based hole transporting material prepared via condensation chemistry as alternative for cross-coupling chemistry for perovskite solar cells
}

\author{
Michiel L. Petrus, ${ }^{1+*}$ Maximilian T. Sirtl, ${ }^{1+}$ Anna C. Closs, ${ }^{1}$ Thomas Bein, ${ }^{1}$ Pablo Docampo ${ }^{2, *}$ \\ ${ }^{1}$ Department of Chemistry and Center for NanoScience (CeNS), University of Munich (LMU) \\ Butenandtstr. 11, 81377 Munich, Germany \\ ${ }^{2}$ Newcastle University, School of Electrical and Electronic Engineering, NE1 7RU Newcastle upon Tyne, \\ UK \\ † These authors contributed equally to this work \\ *e-mail: michiel.petrus@Imu.de,pablo.docampo@newcastle.ac.uk
}

\begin{abstract}
Perovskite solar cells have shown an exceptionally fast increase in performance. So far, most high performing devices comprise expensive hole transport material (HTM) which are synthesized using tedious synthetic procedures, resulting in a high cost and thereby limit the potential use in large-scale applications. In a quest to find low-cost chemistry to link the building blocks, in this work we explore hydrazone-based small molecules. These materials can be synthesized in a simple condensation reaction, with water being the only side product leading to a low material cost of less than $10 \$ / g$. When used as hole transporting layers in perovskite solar cells, highly reproducible performance was obtained, similar to state-of-the-art materials, with the main difference being a small open-circuit voltage loss due to increased interfacial recombination. Thus, we show that hydrazone-based materials have the potential to compete in performance with materials obtained via cross-coupling reactions at a fraction of their cost.
\end{abstract}

Design, System, application

Conjugated hole transporting materials are generally designed by linking building blocks with interesting opto-electronic properties together by using aryl-aryl coupling chemistry. However, these coupling reactions have several disadvantages, such as the inherent formation of side-products, the use of (transition) metal catalysts and their use require stringent reaction conditions, leading to a high product cost. Condensation chemistry offers an excellent alternative to these complicated coupling chemistries, as water is the only side-product and metal catalysts are not required, simplifying product workup and therefore drastically reducing the cost of these materials. In this work we introduce a hydrazone-based small-molecule (EDOT-MPH) as hole transporting material for perovskite solar cells. EDOT-MPH can be synthesized using a simple condensation reaction resulting in an estimated material cost of less than $\$ 10$ per gram. The charge transporting properties of EDOT-MPH are at least as good as those of state-of-the- 
art Spiro-OMeTAD, while the energy levels match with the perovskite. When employed as charge transporting layer in photovoltaic devices very reproducible results are obtained, which approach the power conversion efficiencies of state-of-the-art HTMs in a direct comparison. We demonstrate that hydrazone-based HTM have the potential to compete with state-of-the-art materials in performance at only a fraction of the cost, making this class of materials highly interesting for large scale production.

\section{Introduction}

Perovskite based solar cells are attracting great interest, motivated by the potential to prepare low-cost photovoltaics with excellent performance and the possibility to be flexible and lightweight. However, high quality hole transporting materials (HTMs) leading to record efficiencies are generally synthesized in palladium cross-coupling reactions that require stringent reactions. ${ }^{1-6}$ These chemistries suffer from side reactions and traces of the metal catalyst will stay behind, extensive product purification is required. As a result of the tedious synthesis and workup, not only the cost of these materials is generally high, but it also limits the scalability and impacts the environment as was pointed out by several researchers. ${ }^{1,7-10}$

Recent research mainly focussed on reducing the cost of HTMs by decreasing the amount of synthetic steps. ${ }^{11-13}$ In addition to that, we also focused on drastically reducing the synthetic complexity, cost of materials and the environmental impact of organic HTMs, by moving away from transition metal based cross-coupling reactions. ${ }^{8,9,14}$ Instead, condensation chemistry has been explored, offering an interesting alternative as we showed in our previous work, where we introduced azomethine-based (- $\mathrm{CH}=\mathrm{N}-$ ) HTMs, which were synthesized in a Schiff-base condensation reaction and purified in a simple washing step while the synthesis itself was performed without chlorinated solvents. ${ }^{8,15,16}$ The simple chemistry and product workup resulted in an estimated material costs of $\$ 10$ per gram. ${ }^{8}$ More recent work showed that the cost can be even further reduced to $\$ 4$ per gram by varying the core of the small-molecule HTMs, while these materials have good moisture barrier properties. ${ }^{9}$ Daskeviciene et al. explored condensation chemistry by synthesizing an enamine-based HTM termed "V950", which resulted in impressive power conversion efficiencies of $17.8 \% .{ }^{17}$ Hydrazone-based $(-\mathrm{CH}=\mathrm{N}-\mathrm{NRR}$ ) $\mathrm{HTMs}$ can also be synthesized in a simple and clean condensation reaction, and have shown good performance as electron donor in organic photovoltaics and have potential as hole transporting materials in dye sensitized solar cells with high charge carrier mobilities and tunable thermal properties. ${ }^{18-22}$ However, to the best of our knowledge, these materials have not been examined in perovskite solar cells.

In this work we explore the potential of a hydrazone-based HTM, synthesized using a simple and clean low-cost procedure, in perovskite solar cells and compare the optoelectronic properties to other HTMs prepared via condensation chemistry. We study the charge transporting properties and the effect of conductivity-enhancing additives. Our results show that the hydrazone-based HTM has good charge transporting properties, and shows photovoltaic performance close to state-of-the-art materials when used in planar heterojunction devices. Devices based on hydrazone HTMs have improved reproducibility, demonstrating the potential of simple condensation chemistry for organic electronic applications. 


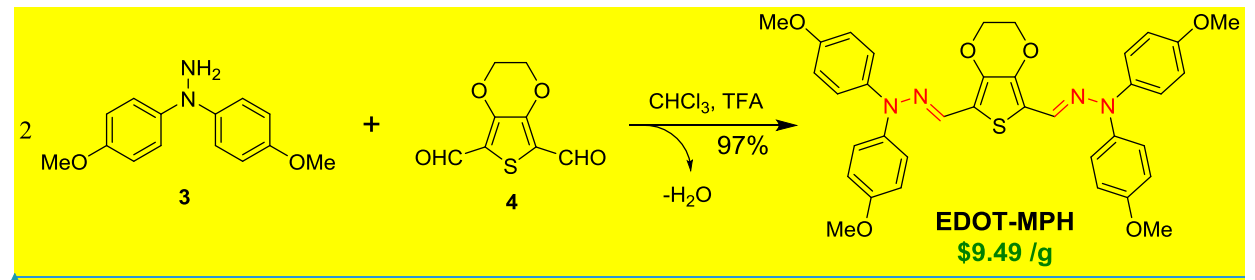

Scheme 1 | Reaction scheme and molecular structure of EDOT-MPH. The hydrazone bond is highlighted and the estimated material cost is shown.

The hydrazine precursor 3 was synthesized following published procedures (Schemes 1 and S1), ${ }^{23-25}$ and was directly used in the condensation reaction with dialdehyde 4, resulting in the hydrazone product (EDOT-MPH) (Figure S2) which was obtained as a bright orange, crystalline powder (Figure S1 and S2) in a near quantitative yield of $97 \%$.

The material cost of the HTM was estimated following a procedure established by Osedach et al. and introduced to the field of HTMs for perovskites by our group. ${ }^{7,8}$ The estimated materials cost on a lab scale is $\$ 9.49$ per gram for EDOT-MPH (details in the supporting information). In comparison, the material cost of Spiro-OMeTAD is estimated to be approximately 10 times higher. ${ }^{8}$ The low cost of EDOT-MPH is ascribed to the simple chemistry that doesn't require expensive metal catalysts and with water as the only side-product, which simplifies the purification of the product. We found that especially purification via column chromatography is very costly. Additionally, as relatively few chemicals are required, this HTM can be considered more environmentally friendly._However, the major environmental concern of perovskite solar cells will be the toxicity of lead, for which several solutions are currently being explored. ${ }^{14,26,27}$ For a better understanding of the environmental impact of organic materials, Sheldon introduced the term $\mathrm{E}$ factor, which is defined as $\mathrm{kg}$ waste per kg product. ${ }^{28}$ For EDOT-MPH we calculated an $E$ value of 400 , while Spiro-OMeTAD has an E factor of 3600 , clearly showing the difference in chemical waste from the synthesis between these materials on a lab scale.

\section{Thermal properties}

As solar cells can reach temperatures up to $80^{\circ} \mathrm{C}$ under operational conditions, it is important that phase transitions of the materials are well above this temperature to ensure a good stability. Therefore the thermal properties were studied by thermogravimetric analysis (TGA) and differential scanning calorimetry (DSC).

The degradation temperature of EDOT-MPH was determined to be $255^{\circ} \mathrm{C}$ (Table 1 and Figure S3), although this is relatively low for HTMs used in perovskite solar cells, it is well above the standard operating temperature of the solar cells. No glass transition $\left(T_{\mathrm{g}}\right)$ was observed by DSC measurements in the temperature range from 0 to $200{ }^{\circ} \mathrm{C}$. While hot-stage microscope measurements revealed a melting transition of the material at $244^{\circ} \mathrm{C}$, which is well above the operation temperature of a solar cell. Apart 
of being a crucial requirement for the materials to be used as an HTM, high melting temperatures are beneficial as they indicate a close molecular packing of the molecule, which has shown to lead to good photovoltaic performance when the materials are used as HTM in perovskite solar cells. ${ }^{9}$

\section{Optoelectronic properties}

A good energy alignment between the highest occupied molecular orbital (HOMO) of the HTM and the valance band of the perovskite is important in order to obtain efficient hole extraction. We determined the HOMO energy from the oxidation onset obtained from cyclic voltammetry (CV) measurements (Figure 2a). The obtained value of $-5.08 \mathrm{eV}$ is slightly higher than Spiro-OMeTAD (Figure $1 \mathrm{~b}$ and Table 1 ). The lowest unoccupied molecular orbital (LUMO) energy level was determined by adding the bandgap to the HOMO energy level and resulted in a value of $-2.7 \mathrm{eV}$. The high LUMO energy level is expected to result in an efficient electron blocking layer.

a)

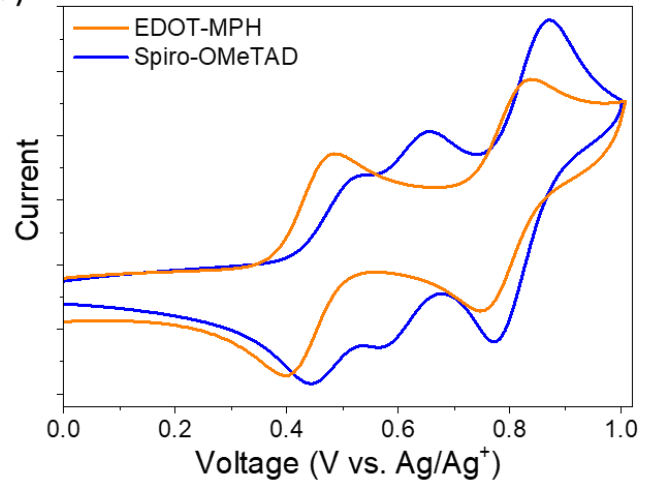

b)

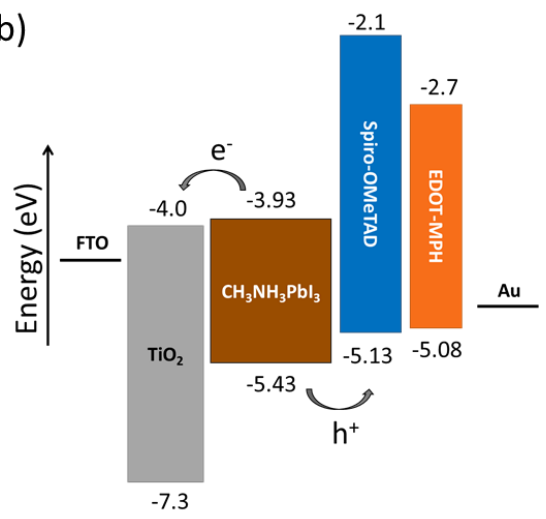

Figure 1 | Electronic properties. a, Cyclic voltammogram of EDOT-MPH measured in a $\mathrm{CH}_{2} \mathrm{Cl}_{2}$ solution containing $1 \mathrm{M} t \mathrm{BuNPF}_{6}$ as electrolyte. $\mathbf{b}$, Energy level diagram showing the energy levels of EDOT-MPH and their alignment with the perovskite.

To gain insights into the geometric and electronic structure of EDOT-MPH, density functional theory (DFT) geometry optimization (B3LYP/6-31G(d,p) level) were performed in vacuum and in dichloromethane (DCM) as the solvent, by means of the conductor-like polarizable continuum model (CPCM) as implemented in the Gaussian 09 program package (Figure 2 and S4). Following the procedure published by Chi et al., ${ }^{29}$ the highest occupied molecular orbital (HOMO) energy level was calculated to be $-5.09 \mathrm{eV}$, which is in excellent agreement with the experimentally obtained value. Figure 2 shows that the HOMO is distributed over the core of the HTM and via the hydrazone bond to one of the phenyl rings on each side. The other phenyl ring is twisted out of the plane of the molecule, which explains why the HOMO is not localized there. The lowest unoccupied molecular orbital (LUMO) is mostly distributed on the core and the hydrazone-bond of the molecule. Additionally, the electron density mapping of the molecules was 
calculated as shown in Figure 2c). The more electronegative part is indicated by the red colour while the more positive parts are blue. The results show a directional dipole within the molecule, that could assist in the formation of a close molecular packing, resulting in good intermolecular charge transport, as was pointed out in our recent work. ${ }^{9}$

a)

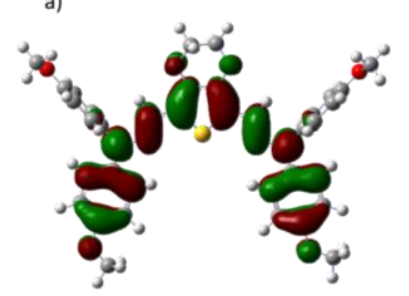

b)

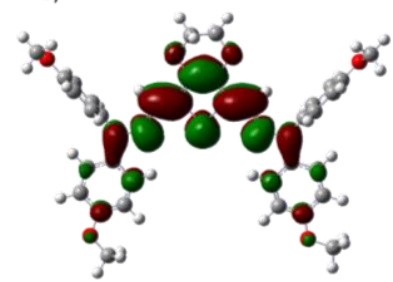

c)

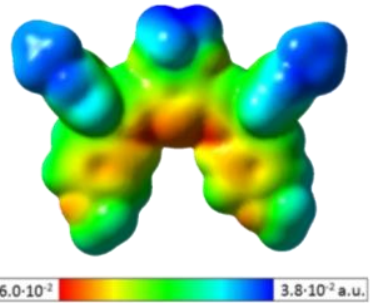

Figure 2 | The optimized molecular geometry and energy level distribution of EDOT-MPH obtained from DFT calculations in DCM. Distribution of the electronic energy levels of a) the HOMO and b) the LUMO orbitals. c) Electrostatic surface potential map, revealing the strong dipole in the core.

Table 1 | Optoelectronic and thermal properties of EDOT-MPH.

\begin{tabular}{lcccccccc}
\hline & $\begin{array}{c}\lambda_{\max } \\
(\mathrm{nm})\end{array}$ & $\begin{array}{c}\lambda_{\text {onset }} \\
(\mathrm{nm})\end{array}$ & $\begin{array}{c}E_{\mathrm{g}} \\
(\mathrm{eV})\end{array}$ & $\begin{array}{c}E_{\text {HOMO, DFT }} \\
(\mathrm{eV})^{\mathrm{a}}\end{array}$ & $\begin{array}{c}E_{\text {HOMO, CV }} \\
(\mathrm{eV})^{\mathrm{b}}\end{array}$ & $\begin{array}{c}E_{\text {LUMO }} \\
(\mathrm{eV})^{\mathrm{c}}\end{array}$ & $\begin{array}{c}T_{\mathrm{m}} \\
\left({ }^{\circ} \mathrm{C}\right)\end{array}$ & $\begin{array}{c}T_{\mathrm{d}} \\
\left({ }^{\circ} \mathrm{C}\right)\end{array}$ \\
\hline EDOT-MPH & 458 & 520 & 2.38 & -5.09 & -5.08 & -2.70 & 244 & 259 \\
\hline
\end{tabular}

a DFT calculation in DCM corrected following the procedure published by Chi et al. ${ }^{29} \mathrm{~b}$ Experimental value obtained from cyclic voltammetry. ${ }^{\mathrm{c}}$ Estimated by adding the optical bandgap to the HOMO energy level. $T_{\mathrm{m}}=$ melting temperature, $T_{d}=$ degradation temperature ( $5 \%$ wt. loss)

\section{Conductivity and mobility}

Lithium bis(trifluoromethanesulfonyl)imide (LiTFSI) is a commonly used additive to assists in the oxidation of the HTM in order to increase to conductivity. ${ }^{30}$ We studied the effect of adding LiTFSI to EDOT-MPH by recording its light absorption spectrum as a function of the additive concentrations (Figure 3). Upon addition of LiTFSI to EDOT-MPH, we observe the formation of one or more new species with absorption maxima at 660 and $740 \mathrm{~nm}$. Upon increasing the LiTFSI concentration, the intensity of the new species increases, while the absorption of the pristine material bleaches. This indicates that EDOT-MPH is oxidized and forms an oxidized or double oxidized species which shows a red shifted absorption profile compared to the pristine material. 

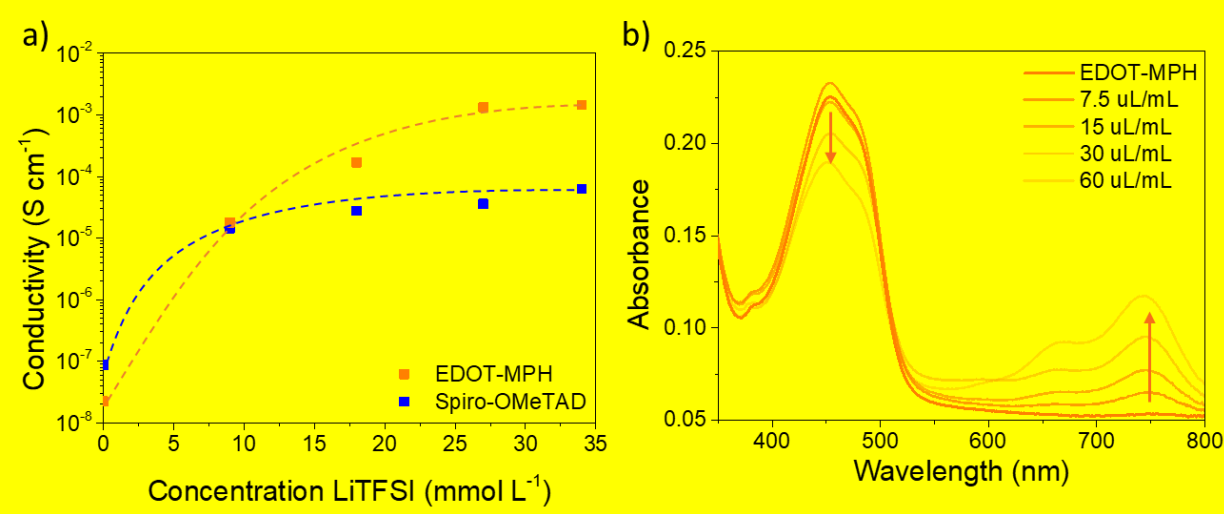

Figure 3 | Charge transporting properties of EDOT-MPH. a, The measured electrical conductivity as a function of the concentration of LiTFSI added to the solution used for spincoating the films. The dotted line is added as a guide to the eye. $\mathbf{b}$, UV-Vis absorption spectra, of EDOT-MPH films as a function of added LiTFSI. The UV-vis spectra show the formation of the oxidized species in the range of $600-800 \mathrm{~nm}$, while the peak of the pristine material bleaches.

In order to characterize the charge transporting properties, hole mobility and conductivity measurements were performed. "Hole-only" devices with a commonly used architecture were prepared to measure the hole mobility (indium-doped tin oxide (ITO)/MoO $/$ EDOT-MPH/MoO $/ \mathrm{Au}$ ). ${ }^{31,32} \mathrm{~J}-\mathrm{V}$ curves were recorded in the dark, with the current assumed to be space-charge limited at higher voltages (Figure S5). From this, the charge carrier mobility can be estimated using the Mott-Gurney equation. EDOT-MPH shows a hole mobility of $2.8 \times 10^{-5} \mathrm{~cm}^{2} \mathrm{~V}^{-1} \mathrm{~s}^{-1}$, which is comparable to the hole mobility measured for pristine SpiroOMeTAD, ${ }^{33}$ and other azomethine- and hydrazone-based HTMs., $22,34,35$

Besides the mobility, the conductivity is an important parameter for charge transport. We measured the conductivity using interdigitating electrodes and studied the effect of oxidizing the HTM with LiTFSI. The initial conductivity is low $\left(2 \times 10^{-8} \mathrm{~S} \mathrm{~cm}^{-1}\right)$, but comparable to Spiro-OMeTAD. ${ }^{30}$ Upon addition of LiTFSI, a clear increase in conductivity is observed which seems to plateau at around $25 \mathrm{mmol} \mathrm{LiTFSI} / \mathrm{L}$ at a conductivity of $2 \times 10^{-3} \mathrm{~S} \mathrm{~cm}^{-1}$. At a LiTFSI concentration of $12 \mathrm{mmol} / \mathrm{L}$ ( which is later used for preparing the hole transporting layer in perovskite solar cells) the conductivity of EDOT-MPH is comparable to that of Spiro-OMeTAD containing $18 \mathrm{mmol}$ LiTFSI $/ \mathrm{L} .{ }^{30}$ Based on these results, a good charge transport to the electrode is expected when EDOT-MPH is used as HTM in a perovskite solar cell, which will minimize the series resistance.

\section{Photovoltaic properties}

The photovoltaic properties of a perovskite device containing EDOT-MPH as HTM were studied by preparing planar perovskite solar cells (Figure 4, S6 and S7) and were tested under ambient conditions. As perovskite absorber, the standard methylammonium lead iodide $\left(\mathrm{MAPbl}_{3}\right)$ and the more recently introduced high performance perovskite $\left(\mathrm{FA}_{0.79} \mathrm{MA}_{0.16} \mathrm{Cs}_{0.05}\right) \mathrm{Pb}\left(\mathrm{I}_{0.83} \mathrm{Br}_{0.17}\right)_{3}$ (FAMACs) were used. ${ }^{36-38}$ The 
deposition of EDOT-MPH was optimized for spincoating from a chloroform/chlorobenzene (1:4 ratio) solution at $40{ }^{\circ} \mathrm{C}$ resulting in a film thickness of approximately $100 \mathrm{~nm}$ (Figure $4 \mathrm{~b}$ ). Similarly to previously studied small molecules accessed via condensation chemistry, also this material shows to have good film forming properties, which allows the preparation of relatively thin homogeneous films. ${ }^{9}$ In contrast, small molecules synthesized via cross-coupling reactions often show their best performance with relatively thick layers $(200-300 \mathrm{~nm}){ }^{6,39}$ which requires more material and thereby further increases the cost. The high quality of our HTM layer furthermore results in a relatively narrow distribution of the photovoltaic performance, especially when compared to Spiro-OMeTAD (Table 2). Additionally, high quality films show an improved stability and due to the lack of pinholes also function as a moisture barrier. ${ }^{9}$

Devices comprising FAMACs as perovskite absorber show a better performance than those with $\mathrm{MAPbl}_{3}$. However, the same trends in photovoltaic performance were observed when EDOT-MPH was used instead of Spiro-OMeTAD (Figure 4, Table S3). With EDOT-MPH as hole transporter, higher FFs are obtained while the $J_{s c}$ is slightly sacrificed. The $V_{\text {oc }}$ however is significantly lower than devices comprising Spiro-OMeTAD. This loss might partly result from the higher HOMO energy level of EDOT-MPH, but could also hint to a higher interfacial charge recombination and/or slow charge carrier injection. ${ }^{40}$ Despite the lower $V_{o c}$, the average PCE of the devices comprising EDOT-MPH is comparable to those with SpiroOMeTAD, and even shows to be slightly higher for EDOT-MPH when combined with MAPbl 3 . Also the stabalized power output shows to be very comparable (Figure S8). The high average PCE is mainly attributed to the narrow distribution in performance when EDOT-MPH is used. 
a)

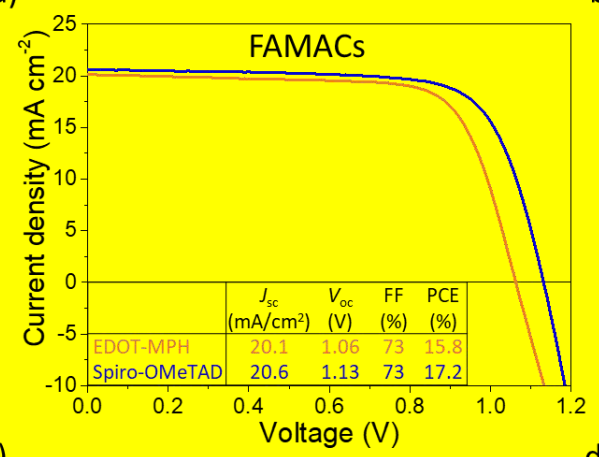

c)

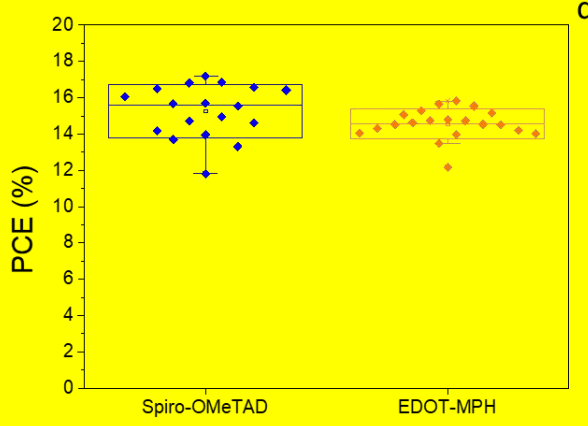

b)

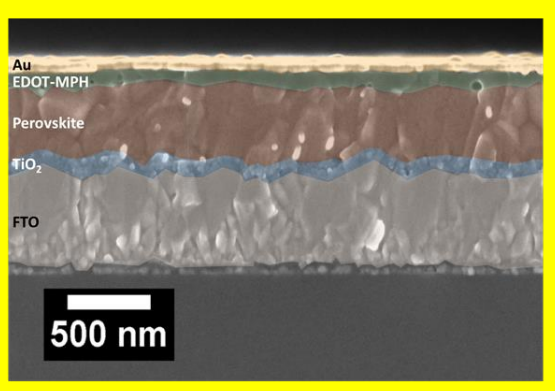

d)

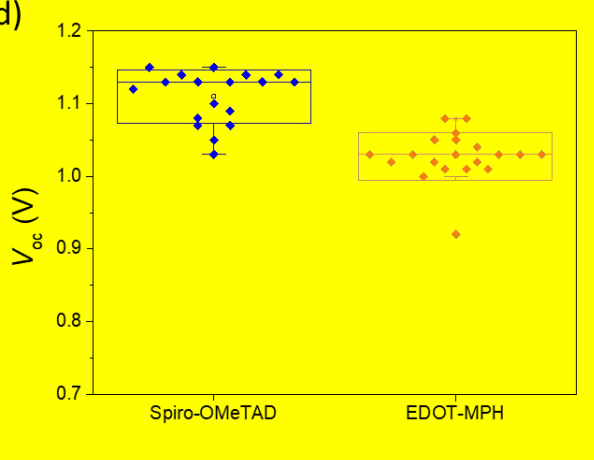

Figure 4 | Photovoltaic performance and reproducibility of FAMACs perovskite solar cells comprising EDOT-MPH or Spiro-OMeTAD as HTM. a, J-V curves collected under AM $1.5 \mathrm{G}$ simulated sunlight of the champion devices comprising EDOT-MPH and Spiro-OMeTAD in the following device architecture: FTO/compact $\mathrm{TiO}_{2} /$ FAMACs/HTM/Au. b SEM cross-section c, $\mathbf{d}$, Box-plot of 38 individual devices prepared showing the comparison of the power conversion efficiency and open-circuit voltage of devices comprising either EDOT-MPH or SpiroOMeTAD as HTM.

Table 2 | Overview of the average device performance prepared using different perovskites.

\begin{tabular}{l|l|llll}
\hline Perovskite & HTM & $\boldsymbol{J}_{\text {sc }}\left(\mathbf{m A} / \mathbf{c m}^{2}\right)$ & $\boldsymbol{V}_{\text {oc }}(\mathbf{V})$ & FF (\%) & PCE (\%) \\
\hline MAPbl $_{3}$ & EDOT-MPH & $19.6 \pm 0.4$ & $1.01 \pm 0.02$ & $68 \pm 6$ & $13.5 \pm 1.4$ \\
MAPbl $_{3}$ & Spiro-OMeTAD & $19.0 \pm 0.8$ & $1.05 \pm 0.04$ & $64 \pm 7$ & $13.2 \pm 1.8$ \\
\hline FAMACs & EDOT-MPH & $19.4 \pm 0.3$ & $1.03 \pm 0.03$ & $73 \pm 2$ & $14.6 \pm 0.8$ \\
FAMACs & Spiro-OMeTAD & $20.8 \pm 0.5$ & $1.11 \pm 0.04$ & $66 \pm 4$ & $15.3 \pm 1.5$
\end{tabular}

Time resolved photoluminescence 
Time resolved photoluminescence (TRPL) has been used to study the lifetime of the charge carriers and their recombination dynamics in a perovskite/HTM bilayer. The decays were acquired using timecorrelated single-photon counting (TCSPC) technique.

Steady state photoluminescence spectra of the $\mathrm{MAPbl}_{3} / \mathrm{HTM}$ films show a single emission peak with a maximum around $770 \mathrm{~nm}$, which is significant lower compared to a pristine $\mathrm{MAPbl}_{3}$ film. Especially EDOTMPH shows a strong reduction of the PL intensity, which indicates a fast hole injection from the perovskite to the HTM and/or significant interfacial recombination.

$\mathrm{PL}$ decay measurements of bilayers consisting of $\mathrm{MAPb}{ }_{3} / \mathrm{EDOT}-\mathrm{MPH}$ and $\mathrm{MAPbl}_{3} / \mathrm{Spiro-OMeTAD}$ were obtained at the same acquisition time (Figure 5 and S9). The initial rise in the PL intensity is slightly lower for EDOT-MPH, which has been linked to a fast hole injection. ${ }^{41}$ However, EDOT-MPH shows a significant faster decay, implying a higher recombination of the charge carriers, which may account for the lower open-circuit voltage and hence lower device efficiency. These results are very comparable to our recently studied azomethine-based HTM, EDOT-OMeTPA, where also a fast charge injection in combination with a high interfacial recombination was observed. ${ }^{40}$

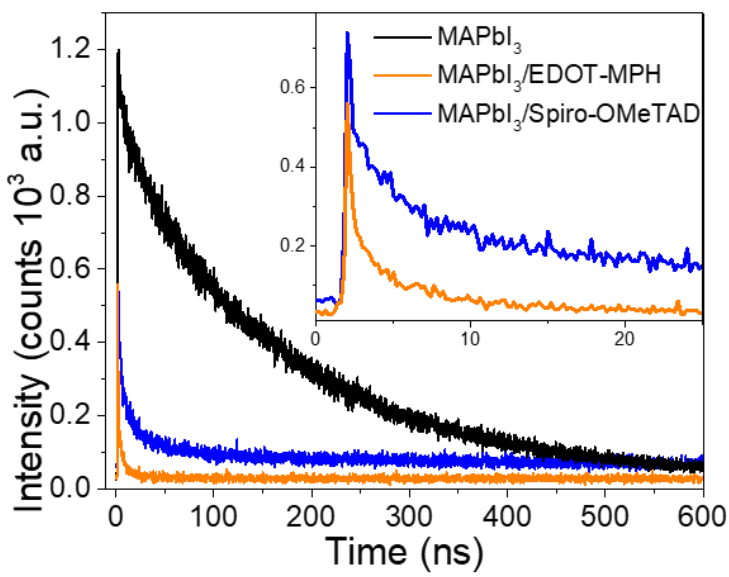

Figure 5 | Time resolved photoluminescence decay of $\mathrm{MAPbl}_{3}$ films and bilayers consisting of $\mathrm{MAPbl}_{3} / \mathrm{HTM}$, obtained for the same acquisition time.

By tuning the molecular structure, the HOMO energy level can easily be fine-tuned, which is expected to result in a higher $V_{\text {oc }}{ }^{9}$ Further tuning of the molecular structure could also improve the interface between the perovskite and the HTM to reduce interfacial charge recombination. By increasing the $V_{\text {oc }}$, it is likely that hydrazone-based materials can outperform devices comprising Spiro-OMeTAD as a result of the good reproducibility. We therefore believe that hydrazone-based materials have a high potential, especially since materials prepared via condensation chemistry can be produced at significant lower cost.

\section{Conclusions}


In conclusion, we introduce a new class of HTMs that are based on a hydrazone-backbone to the field of perovskite solar cells. Our hydrazone-based HTM, EDOT-MPH, is prepared via a simple and clean condensation reaction in which water is the only side product, resulting in a low cost and low environmental impact. EDOT-MPH shows charge transporting properties that compete with current stateof-the-art materials. When applied in perovskite solar cells, a small loss in $V_{\text {oc }}$ is observed which likely originates from an unoptimized energy alignment and interfacial recombination. Nevertheless, the average PCE is comparable to devices comprising Spiro-OMeTAD, mainly as a result of the excellent reproducibility which is linked to the high quality of the HTM layer. These results demonstrate that hydrazone-based HTMs can be produced at a fraction of the cost of current state-of-the-art materials while their performance shows the potential to outperform them.

\section{Synthesis details:}

\section{1,1-bis(4-methoxyphenyl)hydrazine, 3,4-ethylenedioxythiophenedialdehyde}

1,1-bis(4-methoxyphenyl)hydrazine ( $0.33 \mathrm{~g}, 1.35 \mathrm{mmol}, 2.3 \mathrm{eq}$ ) was dissolved in $10 \mathrm{~mL} \mathrm{CHCl}$. To the red solution, 3,4-ethylenedioxythiophenedialdehyde $(0.12 \mathrm{~g}, 0.59 \mathrm{mmol}, 1 \mathrm{eq})$ and one drop trifluoroacetic acid (TFA) were added and the mixture was stirred overnight at room temperature. After that, the reaction was heated to reflux for 4 hours and methanol $(50 \mathrm{~mL})$ was added afterwards, causing a white precipitate and a brown solution. Adding $0.1 \mathrm{~mL}$ trimethylamine (TEA) resulted in an immediate change of the colour of the suspension to bright orange. The product was filtered off as a bright orange powder and dried under vacuum. Yield: $0.37 \mathrm{~g}(0.57 \mathrm{mmol}, 97 \%)$. HRMS (FAB+) m/z: [M] calc. for $\left.\mathrm{C}_{36} \mathrm{H}_{34} \mathrm{O}_{6} \mathrm{~N}_{4} \mathrm{~S}\right)$ 650.2199; Found 650.2224. FTIR: $v\left(\mathrm{~cm}^{-1}\right): 3050(\mathrm{vw}), 3000(\mathrm{w}), 2927(\mathrm{w}), 2831(\mathrm{w}), 1602(\mathrm{~m}), 1548(\mathrm{w}), 1500$ (vs), 1435 (s), 1368 (s), 1299 (m), 1244 (vs), 1199 (vs), 1112 (s), 1065 (vs), 952 (s), 816 (vs). ${ }^{1} \mathrm{H}-\mathrm{NMR}$ (CDCl ; $270 \mathrm{MHz}$ ): $\delta$ $=7.218(\mathrm{~s}, 2 \mathrm{H}), 7.103(\mathrm{dt}, 4 \mathrm{H}, \mathrm{J}=8.8 \mathrm{~Hz}), 6.946(\mathrm{dd}, 4 \mathrm{H}, \mathrm{J}=8.8 \mathrm{~Hz}), 4.086(\mathrm{~s}, 4 \mathrm{H}), 3.831(\mathrm{~s}, 12 \mathrm{H}) \mathrm{ppm} .{ }^{13} \mathrm{C}-$ NMR $\left(\mathrm{CDCl}_{3} ; 101 \mathrm{MHz}\right): \delta=156.60,139.12,137.33,126.31,123.55,115.47,115.05,64.77,55.61 \mathrm{ppm}$.

\section{Acknowledgements}

The authors acknowledge Prof. Theo Dingemans and Dr. Johan Bijleveld for performing the DSC measurements and funding from the German Federal Ministry of Education and Research (BMBF) under the agreement number 03SF0516B, the Bavarian Ministry of the Environment and Consumer Protection, the Bavarian Network "Solar Technologies Go Hybrid", and the DFG Excellence Cluster Nanosystems Initiative Munich (NIM). P. D. acknowledges support from the European Union through the award of a Marie Curie Intra-European Fellowship.

\section{References}

1 M. L. Petrus, J. Schlipf, C. Li, T. P. Gujar, N. Giesbrecht, P. Müller-Buschbaum, M. Thelakkat, T. Bein, S. Hüttner and P. Docampo, Adv. Energy Mater., 2017, 7, 1700264. 
Z. H. Bakr, Q. Wali, A. Fakharuddin, L. Schmidt-Mende, T. M. Brown and R. Jose, Nano Energy, 2017, 34, 271-305.

K. Rakstys, S. Paek, P. Gao, P. Gratia, T. Marszalek, G. Grancini, K. T. Cho, K. Genevicius, V. Jankauskas, W. Pisula and M. K. Nazeeruddin, J. Mater. Chem. A, 2017, 5, 7811-7815.

D. Bi, B. Xu, P. Gao, L. Sun, M. Grätzel and A. Hagfeldt, Nano Energy, 2016, 23, 138-144.

G. Kim, J. Lee, G. Kang, T. Kim and T. Park, Adv. Energy Mater., 2018, 8, 1701935.

J. Zhang, B. Xu, L. Yang, C. Ruan, L. Wang, P. Liu, W. Zhang, N. Vlachopoulos, L. Kloo, G. Boschloo, L. Sun, A. Hagfeldt and E. M. J. Johansson, Adv. Energy Mater., 2017, 1701209.

T. P. Osedach, T. L. Andrew and V. Bulović, Energy Environ. Sci., 2013, 6, 711-718.

M. L. Petrus, T. Bein, T. J. Dingemans and P. Docampo, J. Mater. Chem. A, 2015, 3, 12159-12162.

M. L. Petrus, A. Music, A. C. Closs, J. C. Bijleveld, M. T. Sirtl, Y. Hu, T. J. Dingemans, T. Bein and P. Docampo, J. Mater. Chem. A, 2017, 5, 25200-25210.

W.-Y. Chen, L.-L. Deng, S.-M. Dai, X. Wang, C.-B. Tian, X.-X. Zhan, S.-Y. Xie, R.-B. Huang and L. Zheng, J. Mater. Chem. A, 2015, 3, 19353-19359.

M. L. Petrus, J. Schlipf, C. Li, T. P. Gujar, N. Giesbrecht, P. Müller-Buschbaum, M. Thelakkat, T. Bein, S. Hüttner and P. Docampo, Adv. Energy Mater., 2017, 7, 1700264.

P. Gratia, A. Magomedov, T. Malinauskas, M. Daskeviciene, A. Abate, S. Ahmad, M. Grätzel, V. Getautis and K. M. Nazeeruddin, Angew. Chemie Int. Ed., 2015, 54, 11409-11413.

M. Artiom, P. Sanghyun, G. Paul, K. Ernestas, D. Maryte, K. Egidijus, G. Alytis, J. Vygintas, K. Kristina, C. K. Taek, R. Kasparas, M. Tadas, G. Vytautas and N. M. Khaja, Adv. Funct. Mater., 2018, 28, 1704351.

A. Binek, M. L. Petrus, N. Huber, H. Bristow, Y. Hu, T. Bein and P. Docampo, ACS Appl. Mater. Interfaces, 2016, 8, 12881-12886.

M. L. Petrus, R. K. M. Bouwer, U. Lafont, S. Athanasopoulos, N. C. Greenham and T. J. Dingemans, J. Mater. Chem. A, 2014, 2, 9474-9477.

M. E. Mullholland, D. Navarathne, M. L. Petrus, T. J. Dingemans and W. G. Skene, J. Mater. Chem. C, 2014, 2, 9099-9108.

17 M. Daskeviciene, S. Paek, Z. Wang, T. Malinauskas, G. Jokubauskaite, K. Rakstys, K. T. Cho, A. Magomedov, V. Jankauskas, S. Ahmad, H. J. Snaith, V. Getautis and M. K. Nazeeruddin, Nano Energy, 2017, 32, 551-557.

D. Demeter, S. Mohamed, A. Diac, I. Grosu and J. Roncali, ChemSusChem, 2014, 7, 1046-1050.

S. Urnikaite, T. Malinauskas, I. Bruder, R. Send, V. Gaidelis, R. Sens and V. Getautis, J. Phys. Chem. C, 2014, 118, 7832-7843.

S. Urnikaite, T. Malinauskas, V. Gaidelis, I. Bruder, R. Send, R. Sens and V. Getautis, Chem. - An 
Asian J., 2013, 8, 538-541.

V. Getautis, J. V. Grazulevicius, T. Malinauskas, V. Jankauskas, Z. Tokarski and N. Jubran, Chem. Lett., 2004, 33, 1336-1337.

R. Lygaitis, V. Getautis and J. V. Grazulevicius, Chem. Soc. Rev., 2008, 37, 770-788.

I. A. Tonks, A. C. Durrell, H. B. Gray and J. E. Bercaw, J. Am. Chem. Soc., 2012, 134, 7301-7304.

A. K. Mohanakrishnan, A. Hucke, M. A. Lyon, M. V. Lakshmikantham and M. P. Cava, Tetrahedron, 1999, 55, 11745-11754.

D. Ma, Q. Cai and H. Zhang, Org. Lett., 2003, 5, 2453-2455.

E. Greul, M. L. Petrus, A. Binek, P. Docampo and T. Bein, J. Mater. Chem. A, 2017, 5, 1997219981.

F. Hao, C. C. Stoumpos, D. H. Cao, R. P. H. Chang and M. G. Kanatzidis, Nat. Photonics, 2014, 8, 489.

R. A. Sheldon, Chem. Commun., 2008, 0, 3352-3365.

W.-J. Chi, Q.-S. Li and Z.-S. Li, Nanoscale, 2016, 8, 6146-6154.

A. Abate, T. Leijtens, S. Pathak, J. Teuscher, R. Avolio, M. E. Errico, J. Kirkpatrik, J. M. Ball, P. Docampo, I. McPherson and H. J. Snaith, Phys. Chem. Chem. Phys., 2013, 15, 2572-2579.

M. L. Petrus, F. S. F. Morgenstern, A. Sadhanala, R. H. Friend, N. C. Greenham and T. J. Dingemans, Chem. Mater., 2015, 27, 2990-2997.

D. D. Medina, M. L. Petrus, A. N. Jumabekov, J. T. Margraf, S. Weinberger, J. M. Rotter, T. Clark and T. Bein, ACS Nano, 2017, 11, 2706-2713.

T. Leijtens, I. Ding, T. Giovenzana, J. T. Bloking, M. D. Mcgehee and A. Sellinger, ACS Nano, 2012, 6, 1455-1462.

M. L. Petrus, R. K. M. Bouwer, U. Lafont, D. H. K. Murthy, R. J. P. Kist, M. L. Böhm, Y. Olivier, T. J. Savenije, L. D. a. Siebbeles, N. C. Greenham and T. J. Dingemans, Polym. Chem., 2013, 4, 41824191.

M. Koole, R. Frisenda, M. L. Petrus, M. L. Perrin, H. S. J. van der Zant and T. J. Dingemans, Org. Electron., 2016, 34, 38-41.

Y. Hu, E. M. Hutter, P. Rieder, I. Grill, J. Hanisch, M. F. Aygüler, A. G. Hufnagel, M. Handloser, T. Bein, A. Hartschuh, K. Tvingstedt, V. Dyakonov, A. Baumann, T. J. Savenije, M. L. Petrus and P. Docampo, Adv. Energy Mater., 2018, 8, 1703057.

Y. Hu, M. F. Aygüler, M. L. Petrus, T. Bein and P. Docampo, ACS Energy Lett., 2017, 2, 2212-2218.

M. F. Aygüler, A. G. Hufnagel, P. Rieder, M. Wussler, W. Jaegermann, T. Bein, V. Dyakonov, M. L. Petrus, A. Baumann and P. Docampo, ACS Appl. Mater. Interfaces, 2018, 10, 11414-11419. 
Scopelliti, E. Mosconi, K.-H. Dahmen, F. De Angelis, A. Abate, A. Hagfeldt, G. Pozzi, M. Graetzel and M. K. Nazeeruddin, Nat. Energy, 2016, 1, 15017.

40 E. M. Hutter, J.-J. Hofman, M. L. Petrus, M. Moes, R. D. Abellón, P. Docampo and T. J. Savenije, Adv. Energy Mater., 2017, 7, 1602349.

41 J. Jiménez-López, W. Cambarau, L. Cabau and E. Palomares, Sci. Rep., 2017, 7, 6101.

Table of Content (ToC) Image

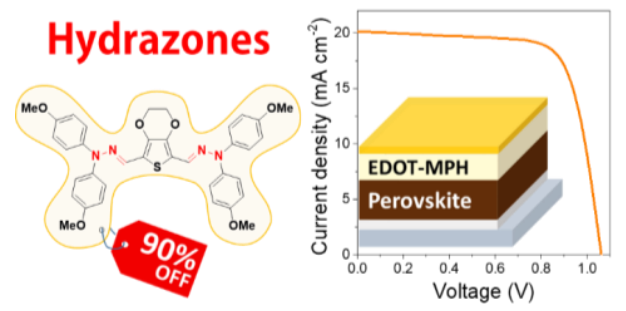

